\title{
Research on the Application of Deep Learning in Artificial Intelligence Courses
}

\author{
Ruijue Wang* \\ KunMing Fenglingjue Technology Co., LTD., Kunming 650000, Yunnan Province, China
}

*Corresponding author: Ruijue Wang, yourtaiyeye@gmail.com

\begin{abstract}
With the in-depth reform of education, taking students as the center, letting students master the basic knowledge of the theory, but also training students' practical skills, is an important goal of the current artificial intelligence curriculum teaching reform. As a new learning method, deep learning is applied to the teaching of artificial intelligence courses, which can not only give play to students' subjective initiative, but also improve the efficiency of students' classroom learning. In order to explore the specific application of deep learning in the teaching of artificial intelligence courses, this article analyzes the key points of the application of deep learning in artificial intelligence courses. In addition, further explores the application strategies of deep learning in artificial intelligence courses. As it aims to provide some useful references to improve the actual efficiency of artificial intelligence course teaching.
\end{abstract}

Keywords: Deep learning; Artificial intelligence courses; Application strategies

Publication date: November 2021; Online publication: November 30, 2021

\section{Introduction}

Compared with shallow learning, in the deep learning mode, students' learning initiative and enthusiasm can be brought into full play, and they can also help students build a systematic knowledge system, which is more helpful for students to understand the theoretical knowledge of artificial intelligence courses and corresponding practical applications. Under the national education policy environment that promotes quality education and focuses on talent training, applying deep learning to artificial intelligence curriculum teaching is an important means to meet the requirements of quality education reform and promote the realization of talent training goals. For this reason, when teachers are teaching artificial intelligence courses, it is necessary to have a deep understanding of deep learning methods and apply them scientifically to classroom teaching. From teaching concepts to learning goals, to classroom models and teaching environments, deep learning is applied in an all-round way. To improve the teaching quality of artificial intelligence courses. Based on this, this article analyzes and explores the application strategies of deep learning in artificial intelligence courses with positive practical significance.

\section{Application points of deep learning in artificial intelligence courses}

\subsection{Based on understanding}

Under the shallow learning mode, students are relatively passive in learning. Normally, teachers will tell theoretical knowledge on the stage, while students will be more passive in accepting knowledge taught by teachers. In this learning process, most students use the traditional learning method, that is, rote memorization, and only a small number of students will memorize on the basis of understanding. In this learning environment, although students understand more theoretical knowledge, their ability to apply knowledge to practice is not high. In the deep learning mode, students turn passive learning into active 
learning. Specifically, on the basis of existing curriculum resources, in addition to imparting basic theoretical knowledge, teachers can guide students to correctly and scientifically understand and master knowledge, which is also an important content of artificial intelligence curriculum teaching under the application of deep learning. Based on understanding, in short, it is to let students abandon the past learning methods of rote memorization, starting from the perspective of understanding, for example, what is the basic connotation? What does the actual application operation look like? Only with a thorough understanding of knowledge can we better grasp knowledge.

\subsection{Build a system of knowledge system}

Constructing a systematic knowledge system refers to combining all the knowledge points together on the basis of understanding and mastering the theoretical knowledge of classroom learning, combining the deep connotation of knowledge, summarizing all the knowledge points, and constructing an overall knowledge framework. With the in-depth reform of quality education, the state is paying more and more attention to educational innovation and development ${ }^{[1]}$. In addition to requiring school teachers to cultivate students' basic literacy, it is also necessary to improve students' practical application ability and promote students' comprehensive development. In order to cultivate students' comprehensive literacy, teachers must not only adopt correct teaching methods to help students master theoretical knowledge, but also allow students to learn to apply theoretical knowledge to solve practical problems. In order to achieve this teaching goal, teachers must apply the deep learning model in depth, first let students briefly understand the knowledge points of artificial intelligence classroom teaching, and then let students understand the knowledge points and master them in a guiding way. On this basis, teachers allow students to try to establish a knowledge framework for students to understand the important and difficult knowledge in artificial intelligence courses from a holistic perspective.

\subsection{Transfer and use of knowledge}

Different from shallow learning, the main purpose of deep learning is to let students know how to use knowledge flexibly in different situations and transform knowledge into practical skills. In the past shallow learning mode, the classroom teaching method is still relatively traditional. Students passively accept knowledge, and most of the time they use rote memorization to acquire knowledge. Although this has certain benefits for students to keep in mind the knowledge points, the key to knowledge learning is to be able to understand it and use it. It is based on this point that the students' understanding of knowledge is not thorough enough. Although they have completed the accumulation of knowledge, students do not fully understand the deep connotation of the relevant knowledge points of artificial intelligence courses. Naturally, the practical application of knowledge is relatively weak, which is inconsistent with the country's requirements for cultivating compound talents. For this reason, when teachers apply the deep learning model in artificial intelligence classroom teaching, in addition to helping students understand key and difficult knowledge, they should also guide students to learn the advancement and application of knowledge. Only in this way can we effectively promote the realization of education goals.

\subsection{Focus on student experience}

Paying attention to student experience is one of the main points in the application of deep learning in artificial intelligence course teaching. It emphasizes that students are the main body first, and then through the construction of corresponding learning situations, students can participate in learning in an experiential way. This will not make students feel boring in the classroom, but can also stimulate students' interest in learning and mobilize students' learning initiative, which can be said to be an important content of the innovative development of artificial intelligence classroom teaching. At present, as the education system 
continues to innovate and improve, school teachers have begun to use new teaching concepts, but because they are deeply influenced by traditional teaching models, they still integrate traditional teaching methods most of the time. Students passively participate in learning activities under the guidance of teachers ${ }^{[2]}$. Although it is different from the past cramming-style teaching, the focus of teaching is still to impart knowledge and does not pay attention to student experience, so it often fails to achieve good teaching results. For this reason, teachers should pay attention to the learning experience when applying the deep learning model in artificial intelligence classroom teaching, so that students can gain knowledge and understand knowledge in the experience, and improve their ability to understand knowledge.

\section{Application strategies of deep learning in artificial intelligence courses}

\subsection{Implement scientific teaching concepts with student experience as the core}

In order to apply the deep learning model to the teaching of artificial intelligence courses, teachers should take student experience as the core and thoroughly implement scientific teaching concepts. By clarifying the specific application methods and approaches of the deep learning model in the teaching of artificial intelligence courses, combined with the basic connotation of deep learning, perfecting teaching goals, choosing appropriate teaching methods, better carrying out artificial intelligence classroom teaching activities, and improving teaching efficiency. First, focus on student experience. The main goal of deep learning is to cultivate students' cognitive and creative abilities. Therefore, teachers must fully mobilize students' learning enthusiasm and initiative in the classroom, which depends on the learning environment and learning methods created by the teacher. By focusing on student experience, choosing appropriate teaching methods and creating a corresponding learning environment, focus on student experience and at the same time give play to the teacher's own guiding role. Second, teachers must implement scientific teaching concepts. With the in-depth reform of education, new educational concepts have gradually penetrated into the education system, and have gradually received national attention and attention. As a trainer of talents in the field of artificial intelligence, teachers should closely follow the pace of development of the times and implement new educational concepts, such as the teaching concept of "leading-subject integration ${ }^{[3]}$." In other words, on the premise of taking students' self-experience as the core, teachers first transfer knowledge to students, and then let students explore knowledge and solve problems autonomously through experience.

\subsection{Develop deep learning goals based on curriculum standards}

Students' deep learning ability is gradually developed through a series of learning processes on the basis of a clear deep learning goal. Therefore, teachers should be aware of the importance of in-depth learning goals, and combine the curriculum standards to formulate in-depth learning goals suitable for students' current learning conditions. Deep learning aims to develop students' analytical and creative abilities, and further promote the improvement of students' comprehensive literacy. In order to effectively achieve this goal, when designing teaching goals, teachers should first start from the student's perspective and understand the knowledge and experience they have in the cognitive structure of students. Based on the requirements of artificial intelligence curriculum standards and students' existing knowledge background, implement the design of class hours or unit teaching goals, and then develop deep learning goals for the fundamental purpose of promoting students' thinking, analysis, and creativity ${ }^{[4]}$. Teachers must not only ensure that the goal of deep learning can effectively promote the cultivation of students' comprehensive quality, but also ensure that the value of the knowledge of artificial intelligence courses can be fully reflected. For example, first understanding the relevant knowledge points of the artificial intelligence course, determine the initial understanding, and then the students personally explore, and finally the teacher will guide the students to summarize and summarize, and improve the students' analytical ability. 


\subsection{Establish a deep learning environment based on intelligent means}

As the country pays more and more attention to the informatization of education, the establishment of a deep learning environment based on intelligent means is an important part of the reform and innovation of artificial intelligence classroom teaching. For this reason, when constructing a deep learning environment, teachers should create a corresponding deep learning environment by using multimedia teaching methods according to the actual student situation of the students and the content of the course knowledge. For example, the establishment of a deep learning environment based on the context of the problem. First of all, teachers are familiar with the knowledge points related to artificial intelligence in this classroom teaching, understand the basic knowledge, important and difficult knowledge points, and ask questions. Secondly, before starting the class, the teacher uses the multimedia to ask the students to briefly understand the knowledge points to be learned in this lesson; then, the teacher asks the students to bring these questions, through independent exploration, cooperation and discussion between students, etc. To find the answer to the question. In this learning process, teachers can act as students, actively participate in student interaction, put forward their own ideas and problem-solving ideas, subtly guide students to think in the right direction, and improve students' problem ability and logical thinking ability. At the same time, teachers instruct students to further memorize knowledge points based on their understanding. Finally, teachers gradually guide students how to use the knowledge to solve practical problems, through exploration and practice, let students master the transfer and application of knowledge, and improve students' comprehensive learning ability.

\subsection{Building a flipped classroom to effectively apply deep learning}

Improving students' deep cognitive ability and creative ability is the main goal of deep learning. For this reason, it is necessary for teachers to adopt a scientific classroom teaching model to apply deep learning in depth. Flipped classroom is a new teaching model that emerged in the development of education informatization. It transfers the traditional teaching habit of "listening to teachers in class and doing homework after class" to the online + offline teaching model, that is, "before class the student watched the teacher's video explanation, then does the homework, (doing experiments) under the teacher's guidance in the classroom or used the knowledge the student learned to solve practical problems." Flipped classroom puts the knowledge transfer process of shallow cognition outside the class, and puts the process of knowledge internalization inside the class. It is also the main goal of deep learning to improve students' deep cognitive ability through teacher-student interaction and mutual exchange and learning between students. For example, before class, teachers can allow students to preview and learn new artificial intelligence courses by themselves through Massive Open Online Courses (MOOC), cloud classroom and other learning apps. During the learning process, the student should record the knowledge points they don't understand and submit them to the teacher before class. In class, after the teacher receives the questions and doubts raised by the students, he organizes the students to carry out practical activities, so that the students can further answer the questions through practice. After the practical activities, the teacher and the students will recall the knowledge points of the whole lesson together and establish an overall knowledge structure to help students consolidate the knowledge points and improve the students' ability to build a systematic knowledge system.

\section{Concluding remarks}

In summary, in the application of deep learning in artificial intelligence courses, the key points are based on understanding, building a systematic knowledge system, transferring and applying knowledge, and focusing on student experience and other elements. Based on this, in the actual application process, teachers should take student experience as the core and implement scientific teaching concepts; set deep learning 
goals based on curriculum standards; build a deep learning environment based on intelligent means; build effective applications in flipped classrooms Deep learning. Due to the limitation of the length of the article, the research on the application of deep learning in artificial intelligence courses in this article is not indepth and complete. In the future, we should continue to pay attention to the relevant research dynamics of deep learning in artificial intelligence courses, and continuously enrich research experience to make up for the deficiencies of this article.

\section{Disclosure statement}

The author declares no conflict of interest.

\section{References}

[1] Wang L, 2021, Exploration of Primary School Artificial Intelligence Teaching Strategies based on Deep Learning. China Modern Education Equipment, (12): 35-36+62.

[2] Wang Y, Wen J, Zhang H, et al., 2021, Research and Practice of the Four-Stage Teaching Method of "Deep Learning" Experimental Teaching. Experimental Technology and Management, 38(05): 208 212.

[3] Zhu W, 2020, Research on Target Detection Cases Based on Python Deep Learning-High School Information Technology School-based Curriculum Artificial Intelligence Case Development. Modern Information Technology, 4(14): 70-72.

[4] Lu B, Zheng Y, Chen Y, et al., 2019, Path Analysis of Deep Learning Courses Under the New Generation of Artificial Intelligence Wave. Computer Education, (10): 151-154+162. 